\title{
Teoria ator-rede e estudos críticos em administração: possibilidades de um diálogo
}

\author{
Actor-network theory and critical management studies: possibilities of a dialogue
}

\author{
Rafael Alcadipani' \\ César Tureta ${ }^{2}$
}

\section{Resumo}

0 objetivo deste artigo é questionar se a teoria ator-rede (TAR) seria, de fato, incapaz de contribuir para análises criticas nos estudos organizacionais. Discutimos, então, as princpais características dos estudos críticos em administração, bem como seu desenvolvimento no contexto brasileiro. Apresentamos ainda alguns dos conceitos iniciais da TAR, que foram alvo de críticas não apenas por naturalizarem as organizações e transmitirem a ideia de que o processo de ordenação é estático, podendo ser entendido de maneira mecânica, mas também por desconsiderarem a dimensão política em suas análises. Como forma de superar tais limitações, que dificultariam um posicionamento crítico da TAR, analisamos os novos desenvolvimentos dessa abordagem, conhecidos como TAR e Depois, que redefiniram algumas de suas principais noções, possibilitando adotar essa perspectiva como "instrumento" analitico critico das organizações. Concluímos que muitas das críticas direcionadas à TAR, no que concerne ao seu caráter acrítico e não-reflexivo, já não se sustentam mais, em função dos novos desdobramentos emergidos da TAR e Depois.

Palavras-chave: estudos críticos em administração; teoria ator-rede; pós-estruturalismo.

\section{Abstract}

The aim of this paper is to questioning whether the Actor-Network Theory would be, in fact, unable to contribute to the critical analyses in organizational studies. We discussed, then, the main characteristics of Critical Management Studies and its development in the Brazilian context. We also present some of the initial concepts of the ANT, which was subject to criticism by naturalizing organizations, transmitting the idea that the ordering process is static and can be understood in a mechanical way, and neglecting the political stance in its analyses. As a way to overcome such limitations, that would make difficult to position ANT as a critical approach, we discuss the new developments of this approach, known as ANT and After, which redefined some of its main concepts, enabling the use of it as a critical analytical "tool" to organizations. We conclude that many of the criticism intended for ANT, regarded to its uncritical and non-reflective character, are no longer appropriate, because of the new developments emerged from the ANT and After.

Keywords: critical management studies; actor-network theory; post-structuralism.

\footnotetext{
${ }^{1}$ Mestre em Administração pela Escola de Administração de Empresas de São Paulo da Fundação Getúlio Vargas (EAESP/FGV), PhD pela Manchester Business School. Professor Adjunto da EAESP-FGV na área de Teoria das Organizações. Endereço: Alameda Lorena, n. 105, apto. 61 - Jardins - São Paulo/SP - Brasil CEP: 01424000. E-mail: rafael.alcadipani@fgv.br.

${ }^{2}$ Mestre em Administração pelo Programa de Pós-Graduação em Administração da Universidade Federal de Lavras (PPGAD/UFLA), Doutorando em Administração pela Escola de Administração de Empresas de São Paulo da Fundação Getúlio Vargas (EAESP/FGV). Endereço: Rua Maracá, n. 132, apto. 124 - Vila Guarani/São Paulo - Brasil - CEP: 04313210. E-mail:cesartureta@gvmail.br
} 


\section{Introdução}

No campo dos estudos da ciência e tecnologia (ECT), a teoria ator-rede (TAR) - também conhecida como sociologia da translação - apresenta-se como uma alternativa às abordagens que focam somente o papel desempenhado pelos humanos ou pelos artefatos, ao analisarem as mudanças e o desenvolvimento tecnológico. Embora tenha origem no campo dos ECT (e.g. EDGE, 1994; MACKENZIE; WAJCMAN, 1999), a TAR tem sido largamente utilizada em outras áreas, como por exemplo, nas pesquisas em estudos organizacionais.

A aplicação e o desenvolvimento da TAR tem gerado debates, ao mesmo tempo em que essa abordagem tem sido alvo de críticas. Walsham (1997) destaca quatro críticas principais: a) análise limitada das estruturas sociais; b) postura amoral ao negligenciar questões de cunho político e moral; c) falha ao considerar a distinção analítica entre humanos e não-humanos; e d) possíveis problemas a respeito de como seguir as entidades numa análise das redes. Embora se considere que, potencialmente, essa abordagem possa dar uma grande contribuição à crítica das organizações, em particular, depois da chamada "virada pós-moderna" no campo dos estudos organizacionais (CALAIS; SMIRCICH, 1999), alguns pesquisadores argumentarm que é falha sua contribuição para o desenvolvimento de abordagens críticas (REED, 1997).

Nesse sentido, ao assumir os novos desenvolvimentos da TAR, também conhecidos pela rúbrica "TAR e Depois" (LAW, 2007; LAW; HASSARD, 1999), assim como o que tem sido considerado as principais características dos ECA, este artigo busca questionar se a TAR seria realmente incapaz de oferecer ideias que possam favorecer o desenvolvimento de uma perspectiva crítica em relação às organizações e contribuir para as pesquisas em ECA.

Para tanto, primeiro, salientaremos os principais elementos daquilo que é conhecido como os ECA de origem anglo-saxã. Faremos isso não como uma forma de assumir ideias estrangeiras de forma acrítica (MIZOCZKY; AMANTINO-DE-ANDRADE, 2005a.), mas, sim, salientar um corpo de estudos que oferece linhas gerais para uma definição do que seria ser crítico em administração. Na sequência, retratarmos a origem e os desenvolvimentos dos ECA no Brasil. Posteriormente, discutiremos algumas das principais ideias presentes nas origens da TAR e que têm sido amplamente empregadas nos estudos organizacionais, contra os quais muitas questões são levantadas, como por exemplo, a de que a TAR oferece uma visão naturalizada dos modos de ordenação estabelecidos, além de não contemplar a dimensão política. Em seguida, a partir de alguns dos recentes desenvolvimentos da TAR e Depois, analisaremos ideias particulares associadas à dimensão política (ontológica) da TAR. Finalmente, apresentaremos nossas conclusões, argumentando que a TAR possui potencial suficiente para contribuir com a análise e reflexão crítica das organizações.

\section{0 que é ser crítico em administração?}

\section{Características dos estudos críticos em administração}

Muito embora os elementos do pensamento crítico estivessem presentes nas ciências sociais desde os anarquistas (e.g. Mikhail Bakunin e Pierre-Joseph Proudhon), os socialistas utópicos (e.g. Henry de SaintSimon e Robert Owen) e os comunistas (e.g. Karl Marx, Friedrich Engels), foi somente nos últimos 40 anos que a análise crítica se desenvoveu nos Estudos Organizacionais (EO) como uma tradição de pesquisa distinta. Sua origem está fundada principalmente nas perspectivas marxistas (e.g. BURAWOY, 1979; BRAVERMAN, 1974; MARGLIN, 1974; THOMPSON, 1989), tendo incorporado mais recentemente algumas ideias pósestruturalistas (e.g. CALÁS; SMIRCICH, 1999; COOPER; BURRELL, 1988).

Nesse contexto, os ECA surgiram nos últimos 15 anos como um movimento, de origem anglo-saxã, que procura envolver diferentes tradições críticas nas mais diversas áreas da administração. Como consequiência, os ECA não constituem um corpo unificado e coerente de conhecimento, devido à diversidade de suas bases epistemológicas, como por exemplo, as variadas formas de marxismo e pós-marxismo (THOMPSON, 1989), a teoria crítica (ALVESSON; WILLMOTT, 1996), o realismo crítico (FLEETWOOD; ACKROYD, 2004), o pós-estruturalismo (CALÁS; SMIRCICH, 1999, as perspectivas feministas (ASHCRAFT; MUMBY, 2004), o 
pós-colonialismo (PRASAD, 2003); o ambientalismo (FORBES; JERMIER, 2002) e a perspectiva foucaultiana (McKINLAY; STARKEY, 1998).

Uma vez que essas tradições seguem diferentes suposições epistemológicas e ontológicas, não há concordância acerca de uma definição precisa sobre o movimento (ADLER, 2002). Em função disso, debates têm sido travados sobre:

- a natureza da crítica nos ECA (e.g. BOJE et al, 2001; CALÁS; SMIRCICH, 1999; HASSARD; HOGAN; ROWLINSON, 2001; JERMIER; CLEGG, 1994);

- se os ECA têm por objetivo gerar práticas administrativas mais éticas e humanas ou se são antiadministração (e.g. ADLER; FORBES; WILLMOTT, 2007; PARKER, 2002);

- sobre a potencial exclusão de outras abordagens críticas por parte dos ECA (veja ACKROYD, 2004; WRAY-BLISS, 2004); e

- sobre as noções estruturalistas e pós-estruturalistas no que tange às questões de poder no ambiente de trabalho (ver PARKER, 1999; THOMPSON; ACKROYD, 1995).

Qualquer definição do que signifique ser crítico em ciências sociais será controvertida. Porém, para Fournier e Grey (2000) e Grey (2005) a pesquisa crítica nos EO se organiza em torno de três proposições centrais: intenções desvinculadas da performance, visão desnaturalizada da administração e reflexividade. Assim, tomando como ponto de partida as ideias de Fournier e Grey (2000), Whittle e Spicer (2008, p.2) destacam que:

[...] a desnaturalização envolve o reconhecimento de que a maneira como as coisas estão não pode ser vista como natural nem inevitável; portanto, pode ser diferente. Segundo, a reflexividade envolve a rejeição da suposição positivista de que a realidade existe "lá fora", aguardando para ser apreendida pelo pesquisador. Ao invés disso, reconhece o papel do analista na construção do conhecimento. Finalmente, uma intenção desvinculada da performance envolve deslocar a ideia da racionalidade meios-fins, que reforça as relações de poder, em direção à consideração de novas formas de ordenamento social.

Nesse sentido, uma das propaladas missões dos ECA é desafiar o caráter opressivo da administração e das organizações (ADLER, 2002; ALVESSON; WILLMOTT, 1992), manter uma postura crítica em relação à razão instrumental (ALVESSON; WILLMOTT, 1996) e opor-se ao poder dominante, à ideologia, ao privilégio administrativo e à hierarquia (GREY, 2005), bem como analisar as relações entre poder e conhecimento, especialmente, mostrando como formas de conhecimento aparentemente neutras, reforçam a assimetria nas relações de poder (ADLER; FORBES; WILLMOTT, 2007; McKINLAY; STARKEY, 1998).

Após essa breve introdução sobre os ECA, na próxima seção traçaremos algumas das características da análise crítica no Brasil, destacando suas peculiaridades e diferenças ante os ECA anglo-saxões, muito embora acreditemos que tais diferenças não devam criar e/ou alimentar barreiras que excluam novas abordagens, as quais, por sua vez, podem lançar diferentes olhares aos aspectos críticos do mundo organizacional no nosso país.

\section{Estudos críticos em administração no Brasil}

A crítica em estudos organizacionais no Brasil remonta às décadas de 1960 e 1970, quando alguns pensadores, como Maurício Tragtenberg, Guerreiro Ramos e Fernando Prestes Motta, já antecipavam muitas das indagações posteriormente levantadas pelos proponentes dos ECA britânicos, no que concerne às formas de dominação e controle que se manifestam nos contextos organizacionais, bem como à necessidade de emancipação dos indivíduos neles inseridos. Como destacam Vieira e Caldas (2006), a contribuição desses autores foi essencial para o início da tradição crítica em administração no Brasil. De acordo com Paes de Paula (2008), a origem dos estudos críticos no Brasil, da qual esses autores fizeram parte, tem no humanismo radical (marxismo e anarquismo) sua fundamentação teórica. Para a autora, isso seria um dos elementos principais a diferenciar os estudos críticos nacionais do movimento dos ECA no exterior, pois este possui um viés preponderantemente (mas, não exclusivamente) pós-estruturalista (veja ALCADIPANI, 2005; MISOCZKY; 
AMANTINO-DE-ANDRADE, 2005a; PAES DE PAULA, 2008). Portanto, a análise crítica das organizações no Brasil possui uma trajetória independente dos estudos nessa perspectiva nos países centrais (PAES DE PAULA, 2008).

Dentre as contribuições dos autores críticos nacionais, podemos destacar a crítica endereçada às teorias administrativas, realizadas por Maurício Tragtenberg. De acordo com esse autor, tais teorias representam, na verdade, uma ideologia (TRAGTENBERG, 1980). Já Guerreiro Ramos se concentrou na crítica à ciência e à pesquisa acrítica pautada em referenciais estrangeiros, que poderiam levar ao que ele denominou de redução sociológica. Além disso, esse autor tratou também das questões relativas à racionalidade substantiva (GUERREIRO RAMOS, 1965, 1989). Prestes Motta, por sua vez, denunciava, sistematicamente, a tentativa das teorias administrativas de fundir a racionalidade instrumental como meio de estabelecer o controle e a dominação, cujo principal objetivo seria permitir que a organização conseguisse maiores lucratividade e produtividade (PRESTES MOTTA, 1979, 1986).

Além de terem deixado diversos seguidores que deram continuidade às suas ideias críticas - ou as tomaram como fonte de inspiração para seus estudos individuais (veja PAES DE PAULA, 2008; VIEIRA; CALDAS, 2006) -, esses autores deixaram ainda um legado crítico ao mundo das organizações, que, hoje, parece estar ganhando destaque novamente na nossa academia.

Em uma pesquisa realizada nos principais periódicos e anais de congressos nacionais, Paes de Paula (2008) identificou que, a despeito de uma recente influência de autores vinculados os ECA britânicos, os autores que fazem crítica em administração no Brasil podem ser divididos em dois grupos: os seguidores das ideias de Guerreiro Ramos e os heterodoxos que se inspiram em Maurício Tragtenberg. Ademais, com base nos dados pesquisados, a autora conclui ainda que, no Brasil, não existem seguidores declarados do movimento dos ECA anglo-saxão, nem dos autores de língua francesa, comumente usados como referência para análises críticas em organizações. Assim, entre os pesquisadores contemporâneos brasileiros, influenciados por Tragtenberg, Guerreiro Ramos ou Prestes Motta, estão José Henrique de Faria, Maria Ester de Freitas, Maurício Serva e Fernando Tenório (PAES DE PAULA, 2008).

Apesar da presença de autores críticos no nosso campo, desde as décadas anteriores, a definição do que é (deveria) ou não ser crítico em administração no Brasil é uma questão que, recentemente, tem levantado algumas polêmicas. Isso decorre da própria dificuldade de se definir o que pode ou não ser considerado uma análise crítica das organizações, bem como sua aplicação no contexto brasileiro. Em debate promovido pela Revista de Administração Contemporânea (RAC), na primeira edição de 2005, Alcadipani (2005), Faria (2005) e Misoczky e Amantino-de-Andrade, (2005a; 2005b) discutem sobre os (des)encontros relacionados à crítica no Brasil e sua possível (in)dependência ou subordinação aos ECA britânicos. Enquanto alguns defendem o distanciamento entre a crítica do movimento europeu (e em grande parte de suas bases epistemológicas) e a crítica nacional (MISOCZKY; AMANTINO-DE-ANDRADE, 2005a; 2005b; PAES DE PAULA, 2008), outros entendem que contribuições de abordagens alternativas com potenciais críticos são sempre bem-vindas (ALCADIPANI, 2005). É dentro desse espírito que elaboramos este artigo.

O crescente interesse pela crítica em administração também pode ser constatado com a criação, em 2007, da linha "Teoria crítica em estudos organizacionais" dentro da Divisão de Estudos Organizacionais (EOR) do Enanpad. No último evento, o painel nacional de EOR foi dedicado à discussão sobre quais as fronteiras (teórico-metodológicas) e o futuro dos estudos críticos em administração no Brasil. Sob a coordenação do professor José Henrique de Faria, participaram do debate os professores Alexandre Faria, Ana Paula Paes de Paula e Maria José Tonelli. Embora os debatedores tenham se posicionado de maneira divergente quanto a algumas questões referentes ao que são os estudos críticos em administração ou teoria crítica em administração, um ponto de concordância foi a necessidade da academia de administração, no Brasil, abrir-se para o debate crítico, de maneira profícua. Além do mais, como muito bem ressaltado naquela ocasião pela professora Maria José Tonelli, precisamos considerar a importância do delineamento dos ECA no Brasil como uma área inclusiva e não, exclusiva, preservando-se, obviamente, o rigor e as fronteiras cambiáveis. Em outras palavras, seria permitir e promover o diálogo entre diferentes abordagens e metodologias que possam suprir limitações 
e/ou falhas presentes em determinadas perspectivas e/ou métodos de pesquisa, para que a própria área consiga se desenvolver de maneira consistente.

Nesse sentido - embora Paes de Paula (2008) ressalte, apropriadamente, a peculiaridade da crítica humanista nacional e argumente em favor da sua preservação, em detrimento da inclusão de perspectivas pósestruturalistas -, acreditamos que a inserção dessas abordagens (pós-analíticas) no desenvolvimento da crítica nacional, não só não abalaria a tradição autônoma da crítica nacional, como também possibilitaria assumir novas óticas de análises capazes de explorar nuanças não apreendidas pelas perspectivas tradicionais. Como destaca Alcadipani (2005), uma das características marcantes dos ECA é sua pluralidade. Ser plural não significa fazer concessões arbitrárias ou praticar a permissividade de forma indiscriminada, mas sim possibilitar, minimamente, que a crítica seja abarcada por perspectivas alternativas e diversas, pois do contrário corremos o risco de substituir uma corrente dominante por outra (veja ALCADIPANI, 2005).

Realizada a discussão sobre o que são os ECA, como eles se desenvolveram e estão se desenvolvendo no Brasil, nas próximos sessões, abordaremos alguns aspectos relacionados com a TAR e o potencial que essa perspectiva tem para contribuir à análise crítica das organizações.

\section{Sociologia da translação e processo organizativo}

Um dos conceitos fundamentais da TAR é a noção de redes heterogêneas (LAW, 1992, 1994), que significa "uma forma de sugerir que a sociedade, as organizações, os agentes e as máquinas são todos efeitos gerados em redes de diversos (não somente humanos) materiais" (LAW, 1992, p.380). Enquanto as entidades, de modo geral, são vistas como estáveis e únicas, a TAR pressupõe que elas sejam o resultado da associação de diferentes elementos heterogêneos (CALLON, 1986; LAW, 1992, 1999a). Nessa concepção, as entidades adquirem seus atributos como conseqüência dos relacionamentos com outras entidades (LAW, 1994, 1999a, 1999b).

Uma vez que as entidades existem em redes de relações (LAW, 1992), os atores e as redes não podem ser concebidos de maneira separada. Por exemplo, todos os atributos normalmente atribuídos aos humanos (pensar, amar, agir etc.) são produzidos em redes compostas por materiais heterogêneos que vão além do corpo (LAW, 1992). Na visão da TAR, os elementos componentes de uma organização são efeitos gerados em múltiplas interações; não, algo dado na ordem das coisas. Nessa direção, as organizações são entidades contínuas e incompletas, precárias e parciais. Analisá-las por meio da TAR é uma forma de tentar compreender por quais meios um sistema difuso e complexo, composto por humanos e não-humanos, se torna uma rede (BLOOMFIELD; VURDUBAKIS, 1999).

A TAR tem utilizado também as noções de móveis imutáveis e de ação à distância para discutir o processo de ordenamento. Móveis imutáveis são formas que possuem a capacidade de "fixar" o conhecimento e permitir que este seja disseminado além do seu ponto de origem (HETHERINGTON, 1997). Os móveis imutáveis tendem a surgir por meio de um longo processo de tradução das informações de interesse (e.g. posição no oceano, tamanho e formato de um território, comportamento de um vírus etc.) em algo imutável e móvel (e.g. mapas, coordenadas espaciais, gráficos etc.), que são os objetos que podem se transportados enquanto mantêm seu formato (LATOUR, 1987, p.227). Para Law (1986), a possibilidade de agir à distância se sustenta no alinhamento de documentos, planos, mapas etc. Tais noções foram discutidas na análise da expansão marítima portuguesa, assim como no trabalho dos cientistas (veja LATOUR, 1987; LAW, 1986), tendo sido desenvolvidas em pesquisas nos EO (veja BLOOMFIELD, 1991). Portanto, assim como os ECA, a TAR, em sua origem, também faz referência a questões relacionadas ao controle.

Ao analisar o processo de ordenação, a TAR desenvolveu a ideia de translação. Esse conceito refere-se "ao trabalho pelo qual os atores modificam, deslocam e transladam seus variados e contraditórios interesses" (LATOUR, 1999b, p.311), na tentativa de torná-los comuns (CALLON, 1986; CALLON; LATOUR, 1981; LAW, 1999a, p.101), o que representa uma das principais ferramentas epistemológicas da TAR para análise do estabelecimento de redes-de-atores (veja CALLON; LAW, 1982; LATOUR, 1987; LAW, 1994). 
Além disso, Callon (1986) oferece - embora, de modo detalhado - uma descrição mecânica desse processo. De acordo com esse autor, a translação é composta por quatro diferentes momentos: problematização, interesse, envolvimento e a mobilização de aliados e estabelecimento de um representante de todos os atores envolvidos numa dada rede-de-atores. Implícita ou explicitamente, vários estudos de caso se basearam nessa ideia de translação, particularmente, no campo de sistemas de informação e tecnologia de informação (e.g. WALSHAM, 1997), e de maneira mais abrangente nos EO (e.g. KNIGHTS; MURRAY; WILLMOTT, 1993).

Entretanto, a descrição detalhada da translação nos termos propostos por Callon (1986) pode caracterizar uma abordagem mais prescritiva do que processual, tendo em vista que propor regras gerais de como a translação ocorre pode parecer uma forma de impor uma maneira particular de como os atores se associam nas redes. Assim, a TAR tem sido acusada de promover uma visão simplista do processo de ordenação, o que poderia afetar a possibilidade desta abordagem possuir um caráter crítico, como veremos a seguir.

\section{Naturalizando as organizações}

As críticas endereçadas à TAR no que concerne a uma suposta visão simplista do processo de ordenação estão relacionadas com a noção de translação, já que esse conceito acaba provendo uma estrutura de análise que pode retratar muitos casos sem qualquer adequação (AMSTERDAMSKA, 1990), constituindo-se em um termo que explicaria praticamente tudo, de vacinas (LATOUR, 1997) a falhas em projetos aéreos (LAW; CALLON, 1988). Como resultado, a TAR parece desconsiderar como translações podem ser distintas e os processos de ordenação apresentarem diferenças e especificidades (AMESTERDAMSKA, 1990).

Ao assumir que as redes-de-atores se tornam irreversíveis, uma vez que a translação ocorra, a TAR tem sido criticada por produzir uma visão determinística das redes (WHITTLE; SPICER, 2008). Ademais, Star e Griesemer (1989) destacam que, ao considerarmos que a translação é "necessariamente descrita do ponto de vista de um ponto de passagem" (STAR; GRIESEMER, 1989, p.390) - e esse ponto é geralmente o do gestor -, esse modelo pode induzir a um viés gerencialista, colocando a TAR em oposição às perspectivas nãogerencialistas e não-performativas como os ECA.

Não é somente a noção de translação que pode levar a uma visão simplista do processo de ordenação. Em algumas análises baseadas na TAR, a ordenação aparece como uma simples maneira de ter uma cadeia efetiva, que seja capaz de transportar os móveis imutáveis e agir à distância, sem, contudo, detalhar as dificuldades e problemas relacionados com a dinâmica do exercício de controle. Uma vez que os móveis imutáveis são gerados, eles parecem permanecer os mesmos, assim como centros e periferias tendem a serem retratados como localidades estabelecidas, negligenciando-se o potencial de resistências e mudanças envolvidas em relações de poder, que são elementos fundamentais na tradição dos ECA.

Outra noção que pode ser vista como formuladora de uma análise simplista do processo de ordenação é a ideia de redes-de-atores, já que esta tem sido desenvolvida de uma maneira rígida, desconsiderando todo o trabalho envolvido na manutenção dessas redes (LAW; SINGLETON, 2005). Nesse sentido, as noções fundamentais da TAR podem levar a uma representação simplista do processo de ordenação, ao mesmo tempo em que as complexidades e diferenças são desconsideradas; algo problemático para qualquer perspectiva crítica que busque explorar toda a complexidade associada com relações que estabelecem ordens (e.g. poder). Consequentemente, algumas críticas acusam a TAR de prover uma análise que naturaliza as organizações (WITTLE; SPICER, 2008). Lee e Brown (1994) afirmam que a TAR se tornou uma formulação metalinguística dentro da qual qualquer sequência de humanos e não-humanos pode ser determinada. Como um sistema totalizante, a TAR não deixa qualquer espaço para a diferença ou não-categorias (HINCHLIFFE Et. Al., 2005, p.676). Ao fazer isso, ela falha na análise da diferença (KIRCH; MITCHELL, 2004), conduzindo a uma problemática visão em termos de política. 


\section{A teoria ator-rede e a sua dimensão política}

Talvez, uma das principais críticas à TAR seja por supostamente desconsiderar a dimensão política (CASTREE, 2002). Amsterdamska (1990) argumenta que a TAR analisa somente a força das alianças que constituem as redes; não, a natureza delas. Na visão da autora, isso significa que para a TAR não importa como as redes se estabelecem: se por meios éticos ou não. Já é destacado que a TAR falha ao definir questões relativas a gênero, raça, classes e colonialismo, comprometendo o estabelecimento das relações que analisa. Star (1991), por sua vez, ressalta que a TAR não reconhece que a heterogeneidade tende a ser diferente entre aqueles que são privilegiados e os que não são; enquanto, para Reed (1997), ela ignora como as oportunidades são desigualmente distribuídas na sociedade. Portanto, a TAR tem sido sistematicamente criticada por ser politicamente neutra, e tais críticas parecem indicar de modo contundente que esta não seria uma abordagem apropriada para o desenvolvimento de uma análise crítica das organizações (veja WHITTLE; SPICER, 2008).

A ausência de engajamento político tem sido discutida pelos principais proponentes da TAR. Law (2007) parece focar mais a ideia de diferença, enquanto Latour (2005a) procura tornar a noção política mais inclusiva. Law (1991b) discute que assumir o relativismo epistemológico não significa compartilhar do relativismo político. Para Law (1997), a política diz respeito à distribuição hierárquica em termos de como o ordenamento cria inclusões e exclusões específicas, que são realizadas de maneira heterogênea (veja LAW, 1997b). Ademais, Law (1999a) salienta como assimetrias podem ser criadas dentro do processo de construção das redes. Entretanto, vale notar que, seguindo as ideias de Foucault, Law (1999b) destaca que embora a ação à distância seja o efeito de uma estratégia de ordenação de elementos, isso não implica a existência de um estrategista consciente; assim como, a qualquer momento, os elementos podem ser ordenados de outra maneira, significando que todas as ordenações poderiam ser diferentes. Isso indica que excluir a TAR como uma possível perspectiva capaz de oferecer contribuições para o desenvolvimento de análises críticas nas organizações é muito mais difícil do que alguns autores indicam (e.g. WHITTLE; SPICER, 2008).

De fato, esses aspectos relacionados com a dimensão política representam um ponto de partida na tentativa da TAR se posicionar de maneira clara em relação a tais questões. Esforços por uma consideração política adquiriram significativo espaço na TAR e Depois. Nesse sentido, se as noções e conceitos iniciais da TAR podem ser vistos como problemáticos para o desenvolvimento de uma análise crítica das organizações, é necessário discutir se o mesmo pode ser aplicado à TAR e Depois.

\section{TAR e Depois: questões sobre ontologia}

As críticas endereçadas à TAR influenciaram novos desenvolvimentos dessa abordagem. Isso é particularmente evidente na discussão associada à TAR e Depois (LAW, 2007; LAW; HASSARD, 1999). Dois dos principais proponentes da TAR (John Law e Bruno Latour) aceitaram o desafio das críticas. Law (1999b) reconhece que a TAR tem privilegiado a estabilidade e a singularidade em detrimento da multiplicidade, ao mesmo tempo em que negligencia aspectos relacionados à diferença. Na tentativa de evitar retratar o processo de ordenação de forma simplista, a complexidade se tornou um objetivo importante para a TAR (HETHERINGTON; LAW, 2000; LAW, 1999a).

De modo geral, os estudos fundamentados na TAR e Depois procuram conferir maior ênfase à dimensão relacional da abordagem como forma de lidar com alguns problemas anteriores. Isso se deu desafiando de maneira mais explícita as tradicionais formas de representação. Latour (1999b) argumenta que a representação é geralmente pensada como algo estabelecido por meio do uso de palavras que expressem o "real". Para esse autor, ao fazer isso, assume-se a realidade como algo que existe "lá fora" e as entidades são vistas como essências dadas na ordem das coisas. Uma das vantagens de seguir o processo de construção é a possibilidade de investigar como se estabelecem conexões entre elementos heterogêneos, como associações se constituem e desfazem, como os elementos se associam e como fatos emergem em função do resultado de tais processos (LATOUR, 2005b). 
Assim, com o intuito de transcender a ideia de representação e tentar encontrar uma descrição para o processo de ordenamento, alguns estudos vinculados à TAR e Depois assumiram a a noção de proposições formulada por Whitehead (LATOUR, 1999c, 2004b).

Proposições não são nem afirmações, nem coisas, nem um intermediário entre ambas. Mais do que isso, são constituídas pela diferença entre essas duas, sem qualquer consideração a priori se tais diferenças são grandes ou pequenas, provisórias ou definitivas, simples ou complexas (veja LATOUR, 1999c, 2004c). Dessa forma, proposições estão relacionadas umas por meio de "articulações", que é um termo que ocupa uma posição neutra nas dicotomias sujeito/objeto e mundo externo e mente, sendo uma propriedade ontológica de diferentes elementos (LATOUR, 1999; 2005). Assim, a questão não é mais se uma afirmação refere-se ou não a um dado estado na ordem das coisas, mas se uma proposição está ou não "bem articulada" (LATOUR, 1999; 2004; 2005b).

Na mesma direção, de acordo com Mol (2002), as entidades são desempenhadas; ou seja, realizadas por meio de práticas materialmente heterogêneas. De fato, o termo "desempenhar" (enact) sugere não apenas que diferentes atividades ocorrem para que algo exista, mas também que a existência está vinculada à ação (MOL, 2002). Mais do que objetos simples que possuem vários atributos ou características, a ideia de "desempenho" salienta que existem diferentes versões da mesma entidade ordenadas como resultado de práticas, representando múltiplas formas da realidade.

Mesmo empregando diferentes terminologias, Latour, Mol e Law reforçam a ideia da TAR de que nada existe $a$ priori e independente das práticas e das cadeias de relações, assim como o processo de ordenação está sempre em processo de produção. Dessa forma, as discussões presentes na TAR e Depois contribuem significativamente para atenuar as acusações de que ela naturaliza os processos organizacionais. Para a TAR e Depois, as entidades, tal como organizações, estão sempre em construção, sem, contudo possuírem qualquer aspecto totalizante e absoluto. A suposta aparência de singularidade, que as entidades podem assumir, são resultados de uma complexa rede de relações e associações que sempre podem ser diferentes (veja JONES; QUATTRONE; McLEAN, 2005; LATOUR, 1997). Czarniawska (2006, p.1557), parafraseando Latour, destaca que "as organizações nunca explicaram nada; são as organizações que precisam ser explicadas". Essa é uma posição que claramente desnaturaliza as organizações, ao invés de tomá-las como certas.

Algumas críticas também foram direcionadas para a dimensão epistemológica da TAR (veja WHITTLE; SPICER, 2008). O argumento é que a TAR falharia em prover uma teoria reflexiva do conhecimento, assim como:

[...] se apoiaria na suposição de que a vida social pode ser observada objetivamente pelos cientistas utilizando conceitos esotéricos, pode ser entendida por meio de processos científicos de verificação e pode ser explicada sem uma avaliação reflexiva das suposições filosóficas e políticas que acompanham o pesquisador [...] [A] TAR tende a impor seu próprio léxico teórico, procura verificar e generalizar um modelo linear e se engaja em uma reflexividade limitada sobre sua própria sustentação de evocação da verdade. (WHITTLE; SPICER, 2008, p.9-10)

O argumento elaborado por esses autores critica a ideia de translação, especialmente, no sentido atribuído por Callon (1986). Com os novos desenvolvimentos da TAR, é possível questionar tais críticas. Primeiro, a TAR e Depois procura abandonar a ideia de translação. Segundo, para a TAR não existe uma dicotomia, a priori, entre pesquisador e objeto, mas ambos são produtos das práticas de pesquisa (LAW, 1997). Por isso, o pesquisador não aparece tendo um comando total do processo de pesquisa e da geração de conhecimento.

Em toda essa discussão, uma das conseqüências do posicionamento da TAR e Depois em relação às questões de representação diz respeito à como essa abordagem lida com as questões da dimensão política, que constituiu um dos seus pontos fracos, conforme destacado anteriormente, mas que foi desenvolvido, adquirindo novo significado. 


\section{Ontologia política da TAR e Depois}

\section{Ontologia política}

Para a TAR, argumentar que a realidade é desempenhada por meio do processo de conhecimento, e que os métodos científicos contribuem para a construção da realidade que se pretende descrever, é mais do que somente uma instância ontológica. Dessa forma, as análises são também políticas (LAW, 2004; 2007; LAW; URRY, 2004), significando que a TAR possui sua ontologia política (LAW, 2004; LAW; URRY, 2004; MOL, 1999, 2002).

A combinação dos termos "ontologia" e "política" sugere que "a condição de possibilidades nunca é dada" (MOL, 1999, p.74-75), mas que está em processo de produção. Dessa forma, as realidades não são imutáveis, são constituídas dentro de nossas práticas mundanas, podendo ser contestadas. Como resultado, a ontologia política refere-se à maneira pela qual a realidade diz respeito à política e vice-versa (MOL, 1999, p.74), significando que as coisas poderiam ser de outra forma. Se se assume que "a desnaturalização envolve reconhecer que a maneira como as coisas são não é nem natural nem inevitável e poderia ser de outra forma" (WHITTLE; SPICER, 2008, p.2), os novos desenvolvimentos da TAR estão alinhados com a dimensão da desnaturalização, que representa um aspecto importante para os ECA.

Ademais, Mol (2002, p.178) discute que, enquanto as entidades são desempenhadas dentro de cadeias de relações específicas, aspectos como diferenças de gênero, de idade, de geração, entre identidade nacional e a condição de refugiado, no âmbito profissional, no que se refere à inteligência emocional e a instabilidades etc. Mais do que separados dos efeitos específicos de ordenação, tais aspectos estão interligados dentro de determinadas realizações. Assim, diferentes realidades interferem uma nas outras criando uma representação bastante complexa.

Outro ponto relevante em relação à ontologia política é se existem opções reais e como elas deveriam ser escolhidas. Mol (1999) discute que diferentes realidades podem incluir umas as outras e que, em alguns casos, elas podem ser conflitantes. Dessa forma, multiplicidade não diz respeito a pluralismo, a entidades separadas que permanecem à parte em campos homogêneos. As várias versões efetuadas possuem relações entre si. Elas podem ser opostas, mas são também complementares. Realidades alternativas podem aflorar;; mas, uma interfere na outra. Separá-las como se fossem plurais significa ignorar as complexas interconexões entre elas. Assim, escolhas racionais em termos políticos não são nem simples de alcançar nem fáceis de escolher (MOL, 1999). Como resultado, a ontologia política está relacionada com o modo como os problemas se constituem (MOL, 2002). De acordo com essa visão, não existem soluções gerais e totais. Elas podem somente ser avaliadas em espaços empíricos locais (LAW, 2007). Contudo, tal como apontado por Law (2007) há diferenças entre as visões políticas da TAR. Na próxima seção, exploraremos as noções de cosmopolíticas.

\section{Cosmopolítica}

Ao questionar as tradicionais formas de representação, a TAR também desafiou a dimensão convencional de política. Geralmente, assume-se que na representação política as entidades e eventos são considerados absolutamente verdadeiros ou falsos (LATOUR, 2003, 2004b, 2005b). Nesse sentido, as decisões políticas são baseadas em "fatos" apresentados como desprovidos de ambigüidades. Por exemplo, durante os eventos que conduziram à invasão e à ocupação militar do Iraque, o secretário de Estados dos EUA proferiu um discurso na Convenção de Segurança das Nações Unidas, argumentando que o Iraque possuía armas de destruição em massa, que poderiam ser facilmente utilizadas. Tais armas dentro do contexto de determinados debates surgiram como fatos, embora em outras articulações tenham surgido como uma controvérsia.

A TAR, portanto, não assume as entidades como singulares e isentas de problemas. Latour (2005b), quando explora as questões relativas à política na TAR, emprega o termo "coisa" (thing) (ou "ding", em alemão), em seu sentido etimológico, para fornecer um significado político diferente a coisas. Etimologicamente, "coisa" refere-se a um tópico central de uma assembleia na qual uma discussão ocorre requerendo o alcance de um julgamento em comum (LATOUR, 2005a, p.250) e designando um tipo de agora arcaica. Para Latour, existem 
muitos outros exemplos de fóruns (e.g. supermercados, instituições financeiras, redes de computadores, laboratórios etc.) que foram excluídos da política moderna. Portanto, a política não se limita à maneira como tem sido usualmente entendida (LATOUR, 2005a; 2007a, 2007b), pois não diz respeito somente ao ato de votar. As ações mais simples, como comprar num supermercado, podem ser vistas como um tipo de voto. O ponto aqui é tentar comparar diferentes técnicas de (re)apresentação, na medida em que existem muitas outras formas de fazer política, que vão além das normalmente consideradas (LATOUR, 2007b).

Essa versão particular da política na TAR objetiva levar em conta a questão da articulação, considerando os participantes que têm sido excluídos pelas tradicionais formas de análises (LATOUR, 2005a). Assim, esse projeto político pressupõe que seja necessário redirecionar a maneira como as entidades são analisadas, buscando reunir os elementos heterogêneos; não apenas os humanos ou os aspectos sociais. Isso demanda o desenvolvimento de uma cosmopolítica. Latour (2004c, p.454) argumenta que nesses termos:

A força de um elemento serve para contrabalancear a força do outro. A presença do cosmos em
cosmopolítica resiste à tendência da política significar o algo exclusivo do mundo dos humanos. A
presença da política em cosmopolítica resiste à tendência de cosmos significar uma lista finita de
entidades que devem ser levadas em consideraçáo. O cosmos protege contra o término prematuro da
política, e a política contra o término prematuro do cosmos. (LATOUR, 2004c, p.454)

A ideia do cosmos é literalmente incluir todas as "coisas" na política, considerando todas as entidades na análise. Ademais, para modificar as condições vigentes, é necessário levar em conta as forças que são produzidas por diferentes conexões nas quais a resistência pode ser aplicada. Se dominação, exclusão, poder etc. devem ser analisados e desafiados, é necessário entender como eles são construídos e geram seus efeitos (LATOUR, 2004c). Nesse sentido, a TAR e Depois confere maior importância às questões políticas, além do entendimento das consequências relacionadas com a performance das realidades. Esse novo desenvolvimento não pressupõe a estabilização das relações, mas procura colocar no centro todas as controvérsias associadas com aquilo que é geralmente considerado um fato.

Assim, críticas endereçadas à TAR, tais como as de que a) essa abordagem distorce o significado da ação política ao elevar o status dos atores humanos, b) evita se comprometer com a emancipação e c) tende a legitimar as relações de poder hegemônicas (veja WHITTLE; SPICER, 2008) se tornam problemáticas quando tomamos como referência a TAR e Depois

No que se refere às relações de poder, alguns proponentes da TAR têm uma visão que se distancia da noção hegemônica. Latour (1986a) argumenta que o problema do poder pode ser descrito por um paradoxo: quando alguém (ou alguma coisa) tem o potencial de poder, nada acontece, mas quando alguém (ou alguma coisa) exerce o poder, de fato, outros desempenham a ação que sempre se transforma enquanto está sendo realizada, a despeito de quem ou o que o exerce. Consequentemente, nenhuma entidade pode exercer poder, a menos que um conjunto de relações seja constituído (LAW, 1991). Para tal visão, a possibilidade do exercício do poder permanece no número de elementos que entram na composição das relações que o estão desempenhando. Assim, a diferença entre o poder potencial e o poder de fato está nos outros, porque o poder só pode ser explicado pelas ações dos outros (LATOUR, 1987. Nessa direção, a TAR assume que o poder só pode ser entendido de maneira relacional; não, total ou absoluta.

A questão da emancipação também tem sido discutida dentro do campo dessa perspectiva. De acordo com Latour (2005a), geralmente, assume-se que os atores estão presos pelo contexto em que se localizam. Como consequência, para libertá-los, é necessário intensificar as ações que se originam "dentro" deles mesmos. O que está por trás dessa ideia é o entendimento de que há nas ações uma essência que tem de ser "salva" / a ser "salva". Entretanto, Latour (2005a) tem uma visão diferente sobre como a emancipação pode ser alcançada. Utilizando a metáfora da marionete, ele argumenta que normalmente assume-se que a dominação é simplesmente transportada do controlador para a marionete, sem transformação. Para emancipar as marionetes, ainda de acordo com Latour (2005b), usualmente, sugere-se cortar todas as linhas que estão prendendo-as. Entretanto, Contudo, / No entanto, o autor argumenta que somente pela multiplicação das conexões com o lado de fora é possível transformar o lado de dentro. Se isso for feito, "o controlador ainda continuará manipulando as cordas, mas cada um dos seus dedos tenderá a mover-se na direção indicada pela marionete. Quanto mais 
cordas a marionete tiver, mais articulada será"” (LATOUR, 2005a, p.187). A ideia aqui é a de que não há nenhuma essência humana a ser libertada, mas a de que humanos são articulados de tal maneira nas relações que, quanto mais relações forem desempenhadas, maiores serão chances de se atingir algum grau de liberdade.

\section{Conclusões}

Tomando como ponto de partida as ideias desenvolvidas pela TAR e Depois e as, geralmente, consideradas principais características dos ECA, este artigo questionou se a TAR seria realmente incapaz de contribuir para uma perspectiva crítica quanto às organizações. Apesar das origens da TAR envolverem noções problemáticas - como translação, móveis imutáveis e redes-de-atores -, sem contar a tendência ao gerencialismo e a negligenciar questões sobre política, novos desenvolvimentos têm buscado superar tais falhas e limitações, visando oferecer uma forma de (ontologia) política da teoria ator-rede. Apesar de indicarmos que as ideias dessa abordagem não podem ser desconsideradas, no que tange a apresentar propostas para uma análise críticas das organizações, cumpre ressaltar que é preciso explorar melhor seu potencial para o nosso campo, especialmente, pela tendência dos estudos organizacionais adotarem a TAR da década de 1990 (cf. LAW, 2007) ou uma de suas antigas versões (cf. LATOUR, 2005a).

Nesse contexto, algumas ideias da TAR podem ser relevantes para tornar explícitas práticas associadas à realização do imperativo do lucro, ao patriarcalismo, à desigualdade racial, a irresponsabilidades ecológicas e ao imperialismo. Da mesma forma, podem ser importantes para evidenciar o conjunto de relações e associações que contribuem para tornar as organizações verdadeiros instrumentos de dominação e exploração. Exploração, dominação, racismo, patriarcalismo, imperialismo etc. não explicam nada, mas são exatamente aquilo que precisa ser explicado (cf. LATOUR, 2004d, 2005b).

Os novos desenvolvimentos da TAR têm potencial crítico, pois reconhecem que as ordens estabelecidas não são inevitáveis e podem ser de outras maneiras, rejeitam as suposições positivistas e não seguem a racionalidade "meio/fim", que reforça o estado estabelecido das coisas. Por outro lado, as ideias associadas à crítica das organizações podem ser muito úteis para a TAR, uma vez que chamam a atenção para as questões negligenciadas por aqueles que recorrem a essa abordagem no nosso campo. 


\section{Referências}

ACKROYD, S. Less bourgeois than thou? A critical review of studying management critically. Ephemera: Theory \& Politics in Organization, v.4, n.2, 2004.

ADLER, P. Critical in the name of whom and what? Organization, v.9, n.3, 2002.

ADLER, P. S., FORBES, L.; WILLMOT, H. On critical management studies. In: BRIEF, A.; WALSH, J. (Ed.). The Annals of the Academy of Management, 2007. Forthcoming.

ALCADIPANI, R. Réplica: a singularização do plural. Revista de Administração Contemporânea, v.9, n.1, 2005.

ALVESSON, M.; WILLMOT, H. Critical management studies. London: Sage, 1992.

;_. Making sense of management. London: Sage, 1996.

AMSTERDAMSKA, 0. Surely you are joking, Monsieur Latour! Science, Technology, \&t Human Values, v.15, n.4, 1990.

ASHCRAFT, K. L.; MUMBY, D. K. (2004). Reworking Gender: A Feminist Communicology of Organization. Thousand Oaks, CA: Sage

BLOOMFIELD, B. P. The role of information systems in the UK National Health Service: action at a distance and the fetish of calculation. Social Studies of Science, v.21, n.4, 1991.

; VURDUBAKIS, T. The outer limits: monsters, actor networks and the writing of displacement. Organization, v.6, n.4, 1999.

BOJE, D. at al. Radicalizing organization studies and the meaning of critique, Ephemera: critical dialogues on organization, v. 08, n. 3 , 2001.

BRAVERMAN, H. Labor and monopoly capital. New York: Monthly Review Press, 1974.

BURAWOY, M. Manufacturing consent: changes in the labour process under monopoly capitalism. Chicago: University of Chicago Press, 1979.

CALÁS, M. B.; SMIRCICH, L. Past postmodernism? Reflections and tentative directions. Academy of Management Review, v.24, n.4, 1999.

CALLON, M. Some elements of a sociology of translation - domestication of the scallops and the fishermen of St-Brieuc Bay. In: LAW, J. Power, action and belief: a new sociology of knowledge? London, Routledge, 1986.

CASTREE, N. 'False Antitheses? Marxism, Nature and Actor-Networks'. Antipode, 34(1): 111-135, 2002.

; LATOUR, B. Unscrewing the big leviathan: how actors macro-structure reality and how sociologists help them do so. In: KNORRCETINA, $K_{;}$CICOUREL, A. (Ed.). Advances in social theory and methodology: toward an integration of micro and macro sociologies. Boston: Routledge, 1981. p.277-303.

; LAW, J. On interests and their transformation - enrollment and counter-enrollment. Social Studies of Science, v.12, n.4, 1982.

COOPER, R.; BURREL, G. Modernism, postmodernism and organization analysis: an introduction. Organization Studies, v.9, n.1, 1988).

CZARNIAWSKA, B. Reassembling the social: an introduction to actor-network theory. Organization Studies, v.27, n.10, 2006.

EDGE, D. The social shaping of technology. In: HEAP, E. et al. Information technology and society: a reader. London: The Open University/Sage Publications, 1994.

FARIA, A. Réplica: ampliando questionamentos sobre crítica em administração._Revista de Administração Contemporânea, v.9, n.1, 2005.

FLEETWOOD, S. ACKROYD, S. Critical Realist Applications in Organization and

Management Studies, London: Routledge, 2004.

FORBES, L. C., ; JERMIER, J. M. 'The institutionalization of voluntary organizational greening and the ideals of environmentalism: Lessons about official culture from symbolic organization theory'. in A. Hoffman and M. Ventresca (eds), Organizations, policy and the natural environment: Institutional and strategic perspectives, 194-213. Stanford: Stanford University Press, 2002.

FOURNIER, V.; LAW, J. AND CALLON, M. 'Engineering and Sociology in a Military Aircraft Project: A Network Analysis of Technical Change'. Social Problems, 35(2): 284-297, 1988.

FOURNIER, V.; GREY, C. At the critical moment: conditions and prospects for critical management studies. Human Relations, v.53, n.7, 2000.

GREY, C. Critical management studies: towards a more mature politics. In: INTERNATIONAL CRITICAL MANAGEMENT STUDIES CONFERENCE, 4., 2005, Cambridge. 
GUERREIRO RAMOS, A. A redução sociológica: introdução ao estudo da razão sociológica. Rio de Janeiro: Tempo Brasileiro. 1965.

A nova ciência das organizações: uma reconceituação da riqueza das nações. Rio de Janeiro: Editora FGV, 1989.

HASSARD, J.; HOGAN, J.; ROWLINSON, M. From labor process theory to critical management studies. Administrative Theory \& Practice, v.23, n.3, 2001.

HETHERINGTON, K. 'Museum Topology and the Will To Connect'. Journal of Material Culture, 2(2): 199-218, 1997.

HETHERINGTON, K.; LAW, J. 'After networks', Environment and Planning D-Society and Space, 18(2): 127-132, 2000.

HINCHLIFFE, S. et al. Urban wild things: a cosmopolitical experiment. Environment and Planning D: Society and Space, v.23, n.3, 2005.

JERMIER, J.; CLEGG, S. Crossroads: critical issues in organization science - a dialogue. Organization Science, n.5, p.1-13, 1994.

JONES, G., C. MCLEAN, ET AL. "Spacing and timing." Organization 11(6): 723-741, 2004.

KNIGHTS, D.; MURRAY, F.; WILLMOT, H. Networking as knowledge work: a study of strategic interorganizational development in the financial services industry. Journal of Management Studies, v.30, n.6, 1993.

LATOUR, B. Science in action: how to follow scientists and engineers through society. Milton Keynes: Open University Press, 1987.

. The pasteurization of France. Cambridge, MA: Harvard University Press, 1997.

On recalling ANT. In: LAW, J; HASSARD, J. Actor network theory and after. Oxford: Blackwell Publishers \& The Sociological Review, 1999a.

Pandora's hope: essays on the reality of science studies. Cambridge, MA: Harvard University Press, $1999 \mathrm{~b}$.

What if we talked politics a little? Contemporary Political Theory, v.2, n.2, 2003.

Politics of nature: how to bring the sciences into democracy. Cambridge, MA: Harvard University Press, 2004a.

Whose cosmos, which cosmopolitics? Comments on the peace terms of Ulrich Beck. Common Knowledge, v.10, n.3, 2004b.

Why has critique run out of steam? From matters of fact to matters of concern. Critical Inquiry, v.30, n.2, 2004c.

From realpolitik to dingpolitik: how to make things public. In: LATOUR, B.; WEIBEL, P. Making things public: atmospheres of democracy. Cambridge, MA: MIT Press, 2005a.

Reassembling the social: an introduction to actor-network-theory. Oxford: Oxford University Press, $2005 \mathrm{~b}$.

Making the 'res public' (interview done by Tomas Sanchez-Criado). Ephemera: Theory \& Politics in Organization, v.7, n.2, 2007a.

Turning around politics a note on Gerard de Vries' paper. Social Studies of Science, v.37, n.5, 2007b.

LAW, J. On the methods of long distance control: vessels, navigation and the Portuguese rout to India. In: LAW, J. Power, action and believe: a new sociology of knowledge? Sociological Review Monograph n.32. Henley: Routledge and Kegan Paul, 1986.

Introduction: Monsters, Machines and Sociotechnical Relations. A Sociology of Monsters? Essays on Power, Technology and Domination. J. Law. London, Routledge: 1-23, 1991.

Notes on the theory of the actor-networking: ordering, strategy and heterogeneity. Systems Practice, v.5, n.3, 1992.

Organizing modernity. Oxford, UK: Blackwell, 1994.

Heterogeneities. 1997. Recuperado em 22 de novembro, 2005 do Centre for Science Studies, Lancaster University, Lancaster LA1 4YN, UK. Disponivel em: <http://www.comp.lancs.ac.uk/sociology/papers/Law-Heterogeneities.pdf>.

After ANT: complexity, naming and topology. In: LAW, J.; HASSARD, J. (Ed.). Actor-network theory and after. Oxford: Blackwell Publishers, 1999a.

Objects, spaces and others. 1999b. Recuperado em 22 de novembro de 2005 do Centre for Science Studies, Lancaster University, Lancaster LA1 4YN, UK. Disponivel em: <http://www.comp.lancs.ac.uk/sociology/papers/Law-Objects-Spaces-Others.pdf>.

After method: mess in social science research. London: Routledge, 2004.

Actor network theory and material semiotics. 2007. Recuperado em 22 de novembro, 2005 do Centre for Science Studies, Lancaster University, Lancaster LA1 4YN, UK. Disponivel em: <http://www.heterogeneities.net/publications/LawANTandMaterialSemiotics.pdf $>$.

;HASSARD, J. Actor network theory and after. Oxford: Blackwell/Sociological Review, 1999. 
; SINGLETON, V. Object lessons. Organization, v.12, n.3, 2005.

; URRY, J. Enacting the social. Economy and Society, v.33, n.3, 2004.

LEE, N.; BROWN, S. Otherness and the actor network - the undiscovered continent. American Behavioral Scientist, v.37, n.6, 1994.

MACKENZIE, D.; WACJMAN, J. The social shaping of technology. Philadelphia: Open University Press, 1999.

MARGLIN, S. What do bosses do? The origins and functions of hierarchy in capitalist production. Review of Radical Political Economics, v.6, n.2, 1974.

McKINLAY; STARKEY, K. Foucault, management and organization theory. London: Sage, 1998.

MISOCZKY, M. C.; AMANTINO-DE-ANDRADE, J. Uma crítica à crítica domesticada nos estudos organizacionais. Revista de Administração Contemporânea, v.9, n.1, 2005a.

$\overline{2005 b .}$

Tréplica: quem tem medo do fazer acadêmico enquanto práxis? Revista de Administração Contemporânea, v.9, n.1,

MOL, A. Ontological politics. a word and some questions. In: LAW, J.; HASSARD, J. (Ed.). Actor-network theory and after. Oxford: Blackwell Publishers, 1999.

The body multiple: atherosclerosis in practice. Durham, NC: Duke University Press, 2002.

PAES DE PAULA, A. P. Teoria crítica nas organizações. São Paulo: Thomson Learning, 2008.

PARKER, M. Capitalism, subjectivity and ethics: debating labour process analysis. Organization Studies, v.20, n.1, 1999.

. Against management. Oxford: Polity, 2002.

PRASAD, A. Postcolonial theory and organisational analysis. Basingstoke: Palgrave Macmillan, 2003.

PRESTES MOTTA, F. C. Controle social nas organizações. Revista de Administração de Empresas, v.19, n.3, 1979.

Organização e poder: empresa, Estado e escola. São Paulo: Atlas, 1986.

REED, M. I. In praise of duality and dualism: rethinking agency and structure in organizational analysis. Organization Studies, v.18, n.1, 1997.

STAR, S. L. Power, technologies and the phenomenology of conventions: on being allergic to onions. In: LAW, J. (Ed.). A sociology of monsters? Essays on power, technology and domination. Sociological Review Monograph n.38. London: Routledge, 1991.

STAR, L.; GRIESEMER, J. 'Institutional Ecology, 'Translations' and Boundary Objects: Amateurs and Professionals in Berkeley's Museum of Vertebrate Zoology, 1907-39'. Social Studies of Science 19(3): 387-420, 1989.

THOMPSON, P. The nature of work. London: Macmillan, 1989.

; ACKROYD, S. All quiet on the workplace front? A critique of recent trends in British industrial sociology. Sociology, v.29, n.4, 1995.

TRAGTENBERG, M. Administração, poder e ideologia. São Paulo: Moraes, 1980.

VIEIRA, M. M. F.; CALDAS, M. P. Teoria crítica e pós-modernismo: principais alternativas à hegemonia funcionalista. Revista de Administração de Empresas, v.46, n.1, 2006.

WALSHAM, G. Actor-network theory and IS research: current status and future prospects. Information systems and qualitative research. In: LEE, A.; LIEBENAU, J.; DEGROSS, J. London: Chapman and Hall, 1997.

WHITLE, A.; SPICER, A. Is actor network theory critical? Organization Studies, v.29, n.1, 2008.

WRAY-BLISS, E. A right to respond? Monopolisation of 'voice' in CMS. Ephemera: Theory \& Politics in Organization, 4, 2004.

\footnotetext{
${ }^{1}$ A TAR utiliza o termo "performance". Entretanto, Mol (2002) afirma que performance remete à ideia de teatro e de coisas que podem acontecer por trás das cenas. Por isso, ela prefere o termo "desempenhar".
} 